\title{
The new candidate IPV6 address size prediction
}

\author{
Khaldoun Batiha \\ Dean of Faculty of Information and Technology, Philadelphia University, Jordan \\ E-mail:kh_batiha@philadelphia.edu.jo
}

Copyright $\odot 2014$ Kaldoun Batiha. This is an open access article distributed under the Creative Commons Attribution License, which permits unrestricted use, distribution, and reproduction in any medium, provided the original work is properly cited.

\begin{abstract}
IPv4 protocol is now no more sufficient due to its limited address space where this protocol uses only 32-bit for addressing. IPv6 protocol is the next protocol which was introduced as a new protocol that provide a huge address space .In This paper we discussed the significant overhead in the IPv6 standard packet due to its 128 bits address size, so we develop three different studies in order to generate a prediction of exhibition date for several address sizes so we can suggest another size for address size in IPv6 to improve the overall performance of the internet and tolerate the overhead by reducing the address size. In the same time this size should accommodate the accelerated growth in needs for unassigned blocks of addresses for very long time.
\end{abstract}

Keywords: Ipv4 Protocol, Ipv6 Protocol, Addressing Types, Ipv6 Header, Exhibition Date, Address Pool.

\section{Introduction}

Nowadays, IPv4 protocol is facing extremely serious problem due to its limited address space where this protocol uses only 32-bit for addresses which in few years will be no more sufficient to accommodate the accelerated growth in needs for unassigned blocks of IPv4 addresses (known as exhibition problem)[1,2,3]. Meanwhile, IPv6 was introduced as a life saver for expected exhibition problem by expanding address IP address space beyond all expected internet and application Escalated needs in future. In fact IPv4 address space multiplied by $2^{96}$ produces a huge address space using 128-bit address size.

This paper spots a significant overhead in the IPv6 standard packet where the address occupies $80 \%$ of the packet header. So we developed three different studies in order to generate a prediction of exhibition date for several address sizes so we can suggest another size for addresses in IPv6 to improve the overall performance of the internet and tolerate the overhead by reducing the address size.

The first study was made depending on consumption rate which was developed by Geaff Haston, It discusses the predicted exhaustion dates of unallocated IP addresses for size range between 32-bit and 64-bit in the internet. The unallocated address pool exhaustion for this range with size interval of 4-bit is discussed using a study that we develop on some basic rules taken from Geaff Haston study of ipv4 utilization and consumption in 2005 [1,2]. The study predicts when the unallocated address pool will be exhausted and no more IP addresses will be available. On the other hand, it shows which address size will solve the exhaustion problem. At the same time, this size should be less than 128-bit, otherwise this study will be useless since its main goal is to reduce the current IPv6 address size.

The second study depends on a short-term projection for World Population numbers issued by international programs [4]. It also discusses the predicted exhaustion dates of unallocated IP addresses for size range between 32-bit and 128bit in the internet. The unallocated address pool exhaustion for this range with size interval of 8-bit is discussed using the study that we develop based on IPv4 address report generated at 30-Jan-2013 [1] the remaining addresses in RIR pool and world population projection growth rate including the derivation of new formula that predicts IP Address consumption. On the other hand, it shows which address size will solve the exhaustion problem. At the same time, this size should be less than 128-bit, to reduce the overhead in IPv6 packet basic header by $40 \%$.

Finally, we used both studies to develop a third one which depends on both studies by generating a table that reflects a variable effect for each study in each row, and then we generate the average of overall generated effects. 


\section{IPv6 Overview}

In general, IP is a network layer protocol used to route data through a number of networks until it reaches the local network for destination host. Where each host has its own IP address either IPv4 address or IPv6 address. In particular cases, a host could have both IPv6 and IPv4 address (dual stack host), also each network has its own address. IPv4 address is divided into 4 bytes used to represent the network part and host part of the IP address using notation called dotted decimal notation, while IPv6 is much larger[5,6,7]. It is divided into eight 16-bit words represented by 32 hexadecimal digits.

Internet Protocol version 6 (IPv6) is an Internet Protocol version which is designed to succeed IPv4. In general IP is a network layer protocol used to route data through a number of networks until it reaches the local network for destination host. Where each host has its own IP address either IPv4 address or IPv6 address or a host could have both IPv6 and IPv4 addresses (dual stack host). Also each network has its own address. IPv4 address is divided into 4 bytes used to represent the network part and host part of the IP address using notation called dotted decimal notation[5,7], while IPv6 is much larger. It is divided into eight 16-bit words represented by 32 hexadecimal digits.

IPv6 was defined in December 1998 by the Internet Engineering Task Force (IETF). The first implementation which is still in dominant use currently. It is an Internet Layer protocol for packet-switched internetworks. It also implements new features that simplify aspects of address assignment (stateless address autoconfiguration) and network renumbering (prefix and router announcements) when changing Internet connectivity providers. The IPv6 subnet size has been standardized by fixing the size of the host identifier portion of an address to 64 bits to facilitate an automatic mechanism for forming the host identifier from Link Layer media addressing information (MAC address). IPv6 provides several services and capabilities that make IPv6 a superior internet protocol like plug-and-play auto configuration, Quality of service flow label [3,7], multicasting, simplified processing by routers, only permit source host pay load fragmentation, multicast lisener discovery messages, ICMPv6 Router Solicitation and extension headers.

\section{The role of IPv6 in solving IPv4 exhibition problem}

The main reason for introducing IPv6 is to overcome the concerns of the rapid growth in internet which demands more and more unallocated ip addresses to be available to accommodate this growth. IANA IPv4 unallocated address pool exhaustion will be very soon [1,2]. Since the address space that IPv4 provides is no more sufficient for the internet needs today, where many studies predict that the A platform to provide flexibility for further growth and expansion has become urgent. And the main feature of this platform should be the address space size that it represents.

IPv6 was present as a life saver for IPv4 exhaustion problem since it supports 128 bit IP address. Sixteen bytes or 128 bits can accommodate 340,282,366,920,938,463,463,374,607,431,768,211,456 IPv6 addresses which mean that there are enough IPv6 addresses for every portion in the universe [5,7]. This address space will not face the exhaustion problem even after a billion years.

\section{The overhead of IPv6 basic header}

If we look at IPv6 packet header in figure1, we can see clearly that IPv6 source and destination IP address occupy 32 bytes from 40 bytes of the basic IPv6 header total size (Figure1), which means $80 \%$ of header total size. This is considered a big overhead in comparison with the basic header total size [8]. So in order to make an effective reduction in IPv6 packet overall overhead, a reduction in IPv6 address size is the ideal solution. But the question here is: how much should the reduction be? To answer this question, we need to take into consideration that the new address size should be large enough so that it can accommodate the needs of the world in the far future.

\section{IP Address exhibition prediction studies}

In this paper we developed three different studies in order to generate prediction for IP address exhibition date for several address sizes. The main questions that we need to answer in each study are: when will remain unallocated addresses of RIR be exhausted? And if we have the choice to increase the size of IPv4 address, what is the most effective size? Is the IPv6 address size the most effective size? In order to answer these questions, we need to predict the exhibition date for several IP address sizes and then try to choose the smallest size with acceptable exhaustion date.

\subsection{IP exhibition prediction first study}

This study will depend on the address consumption rate which was developed by Geaff Haston. According to Geaff Haston the IPv4 utilization and consumption in 2005 are as follows: $14 \%$ of 256/8 blocks is reserved and the remaining $220 / 8$ blocks or $86 \%$ of total IPv4 address space are available for general use. $66 \%$ of this available address space was allocated in 2005 which means the available address space for allocation in 2005 was 74/8 blocks. The consumption 
rate in 2002 was 4/8 blocks per year and it increased to reach 6/8 blocks per year in 2005 [2]. This growth in consumption rate in three years led to an increase in consumption rate equal to $1.5 / 8$ blocks per year.

32 bits are equivalent to $256 / 8$ blocks of ip address space that means if we use 33 bits in ip address, this will be equivalent to 512/8 blocks. In Table 1, we have listed the equivalent number of / 8 blocks for the range of ip size in bits (32 bits to 64 bits). We have also listed the expected unassigned address space for each size in 2005 . We have picked this year since this is the year of Geaff Haston study which will be used as a base for our prediction for ip address exhibition. That means the values in the unassigned ip address column represent the remaining ip addresses blocks in 2005 if we use the corresponding ip address size $\mathrm{N}$.

Now we will use the Geaff Haston study consumption rate and increase consumption rate per year to predict the exhaustion of the available address space for an IP address range from 32-bit to 64-bit. (For example the study predicts that unassigned address for the 32 bit address space will be exhausted by 2012). In the same way, we will predict when the address space for each ip address size will be exhibited. To cover the range from 32bit to 64bit, we pick an interval of 4 bits length between two consecutive addresses size. Figure 2 shows the prediction for this range of addresses size.

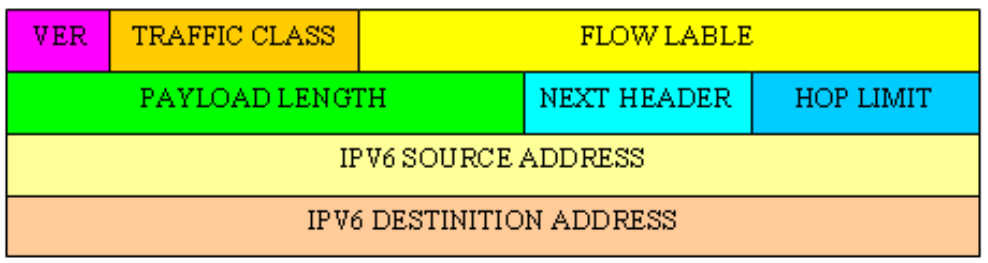

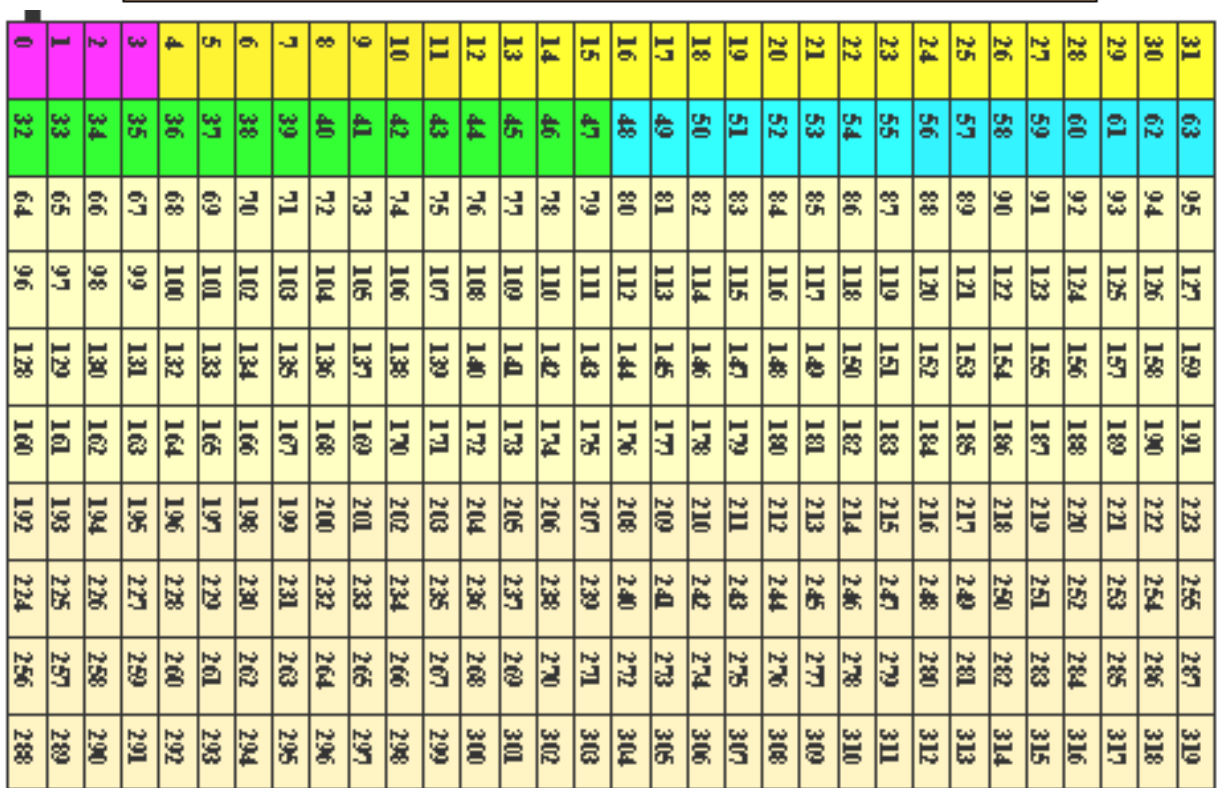

Fig. 1: 320-bits IPv6 basic header: each color represents part of the header so the first part of the figure shows the names of IPv6 parts while the second part shows bits numbers for each part, (VER $\rightarrow 0-3$, TRAFFIC CLASS $\rightarrow$ 4-11, FLOW LABLE $\rightarrow$ 12-31, PAYLOAD LENGTH $\rightarrow$ 32-47, NEXT HEADER $\rightarrow$ 48-55, HOP LIMIT $\rightarrow$ 56-63, IPV6 SOURCE ADDRESS $\rightarrow$ 64 $\rightarrow$ 191, IPV6 DESTINITION ADDRESS $\rightarrow$ 192 $\rightarrow$ 319)

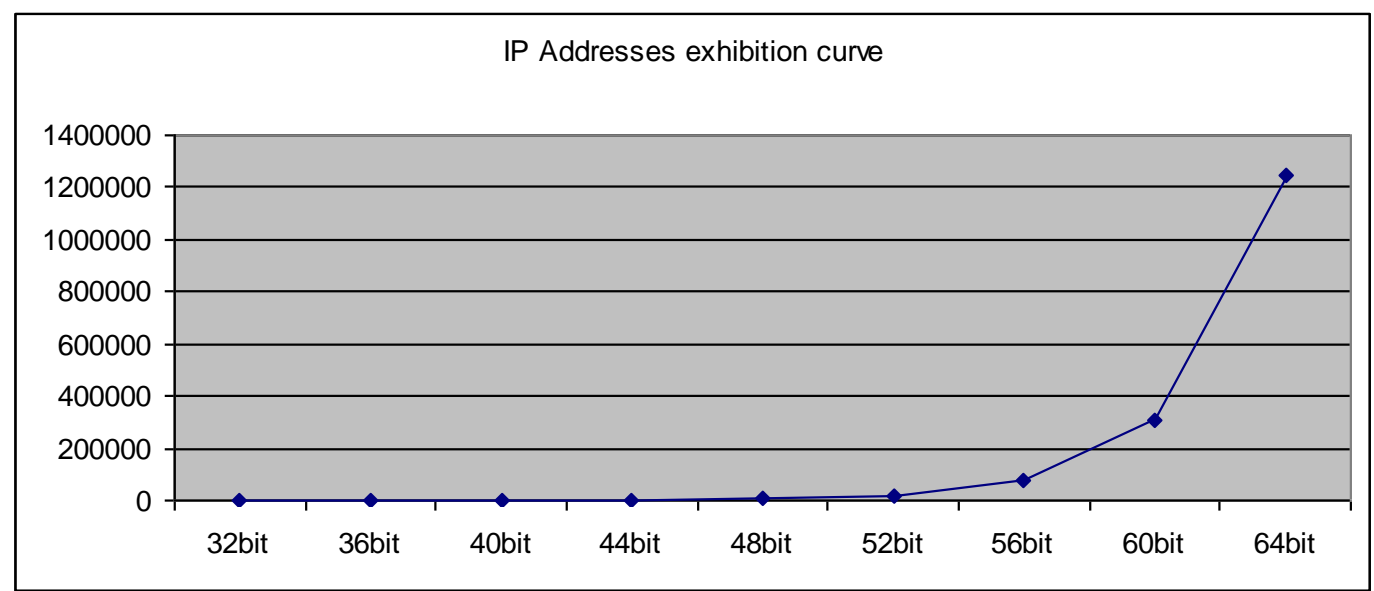

Fig .2: Curve represent the relation between the number of years that range between 32-bit and 64-bit of IP address can accommodate which grows up exponentially starting from year 2012 for address size 32-bit until the year of 1244149 for address size 64-bit with difference equal to 1242137 years 
As we can see in Figure 2, the number of years that range between 32-bit and 64-bit of IP address can accommodate is growing up exponentially starting from year 2012 for address size 32-bit until the year of 1244149 for address size 64 bit with difference equal to 1242137 years.

For IP address size 36-bit the exhibition date will be in year 2074, and for the next ip address size in the figure the date becomes 2296. After that, the exhibition date that 44-bit address size will offer is 3183 which means 887 years more than the previous one and this number increases for 48-bit address size to 3548. Then, for 52-bit address size the exhibition date will be 230920 and for 56-bit it will be 77675 which will be increased by 227015 years for 60 -bit address size. Finally, for 64-bit address size the exhibition date will jump all the way to 1244149 .

If we reduce the IPv6 address size to 64-bit. This size can accommodate: 18446744073709551616 IPv6 addresses and exhibition date will be at year 1244149 , which is more than enough. So why should we stick to ip address size of 128 bit while 64-bit address size provides reduction of basic header IPv6 packet overhead up to $40 \%$ of the packet header total size which is considered as a significant reduction, that will improve the overall IPv6 performance.

Table 1: Equivalent number of / 8 blocks for the range of ip size in bits (32 bits to 64 bits), and the expected unassigned address space for each size in 2005

\begin{tabular}{|c|c|c|}
\hline $\mathbf{N}$ & /8 Blocks & Unassigned /8 Blocks \\
\hline 32 & 256 & 74 \\
\hline 33 & 512 & 330 \\
\hline 34 & 1024 & 842 \\
\hline 35 & 2048 & 1866 \\
\hline 36 & 4096 & 3914 \\
\hline 37 & 8192 & 8010 \\
\hline 38 & 16384 & 16202 \\
\hline 39 & 32768 & 32586 \\
\hline 40 & 65536 & 65354 \\
\hline 41 & 131072 & 130890 \\
\hline 42 & 262144 & 261962 \\
\hline 43 & 524288 & 524106 \\
\hline 44 & 1048576 & 1048394 \\
\hline 45 & 2097152 & 2096970 \\
\hline 46 & 4194304 & 4194122 \\
\hline 47 & 8388608 & 8388426 \\
\hline 48 & 16777216 & 16777034 \\
\hline 49 & 33554432 & 33554250 \\
\hline 50 & 67108864 & 67108682 \\
\hline 51 & 134217728 & 134217546 \\
\hline 52 & 268435456 & 268435274 \\
\hline 53 & 536870912 & 536870730 \\
\hline 54 & 1073741824 & 1073741642 \\
\hline 55 & 2147483648 & 2147483466 \\
\hline 56 & 4294967296 & 4294967114 \\
\hline 57 & 8589934592 & 8589934410 \\
\hline 58 & 17179869184 & 17179869002 \\
\hline 59 & 34359738368 & 34359738186 \\
\hline 60 & 68719476736 & 68719476554 \\
\hline 61 & $1.37439 \mathrm{E}+11$ & $1.37439 \mathrm{E}+11$ \\
\hline 62 & $2.74878 \mathrm{E}+11$ & $2.74878 \mathrm{E}+11$ \\
\hline 63 & $5.49756 \mathrm{E}+11$ & $5.49756 \mathrm{E}+11$ \\
\hline 64 & $1.09951 \mathrm{E}+12$ & $1.09951 \mathrm{E}+12$ \\
\hline
\end{tabular}

\subsection{IP exhibition prediction second study}

The ipv4 can provide address space up to $256 / 8$ blocks, $14 \%$ of these blocks are reserved and the remaining (86\%) are available for allocation. So $220 / 8$ blocks is the total address space available for allocation. According to IPv4 address report generated on 30-Jan-2013 [1], the remaining addresses in RIR pool (/8s) are as follows: for APNIC is 0.8905/8 block, for RIPE NCC is $0.9357 / 8$ block, for ARIN is $2.9634 / 8$ block, for LACNIC is $2.6080 / 8$ block and for AFRINIC is 3.8019/8 block which means that the total remaining addresses in RIR pool is $11.1995 / 8$ block.

IPv4 addresses exhaustion date is the time when the pool of available addresses in RIR reaches the last /8 block available for allocation. In other words, if the remaining unallocated addresses for RIR address pool are less than 
$16,777,216$ addresses . From what has been stated above, we can conclude that the total allocated RIR address space of IPv4 in 2013 is $209.7225 / 8$ blocks.

\subsubsection{Available address space for IP address of size $N$}

As we mentioned before, IPv4 address are equivalent to 256/8 blocks of IP address space. That means if we use 33-bits in IP address, this will be equivalent to $512 / 8$ blocks. In Table 1 we listed the equivalent number of $/ 8$ blocks for the range of IP size in bits (32-bits to 128-bits). We also listed the expected unassigned address space for each size in 2013. We have picked this year since this is the year of IPv4 address report generated (30-Jan-2013) [1] for remaining addresses in RIR pool (/8s) which will be used as a base for our prediction for IP address exhibition date. That means the values in the unassigned IP address column represent the remaining IP address blocks in 2013 if we use the corresponding IP address size $\mathrm{N}$.

Table 2: The equivalent number of $/ 8$ blocks is listed for the range of IP size in bits (32-bits to 128-bits) together with the expected unassigned address space for each size in 2013.

\begin{tabular}{|c|c|c|c|c|c|}
\hline IP Address Size (N) & Unassigned /8 blocks & $\begin{array}{l}\text { IP Address } \\
\text { Size }(N)\end{array}$ & $\begin{array}{l}\text { Unassigned /8 } \\
\text { blocks }\end{array}$ & $\begin{array}{l}\text { IP Address } \\
\text { Size }(\mathrm{N})\end{array}$ & $\begin{array}{l}\text { Unassigned /8 } \\
\text { blocks }\end{array}$ \\
\hline 32 & 11 & 65 & $2.19902 \mathrm{E}+12$ & 98 & $1.88895 \mathrm{E}+22$ \\
\hline 33 & 267 & 66 & $4.39805 \mathrm{E}+12$ & 99 & $3.77789 \mathrm{E}+22$ \\
\hline 34 & 779 & 67 & $8.79609 \mathrm{E}+12$ & 100 & $7.55579 \mathrm{E}+22$ \\
\hline 35 & 1803 & 68 & $1.75922 \mathrm{E}+13$ & 101 & $1.51116 \mathrm{E}+23$ \\
\hline 36 & 3851 & 69 & $3.51844 \mathrm{E}+13$ & 102 & $3.02231 \mathrm{E}+23$ \\
\hline 37 & 7947 & 70 & $7.03687 \mathrm{E}+13$ & 103 & $6.04463 \mathrm{E}+23$ \\
\hline 38 & 16139 & 71 & $1.40737 \mathrm{E}+14$ & 104 & $1.20893 \mathrm{E}+24$ \\
\hline 39 & 32523 & 72 & $2.81475 \mathrm{E}+14$ & 105 & $2.41785 E+24$ \\
\hline 40 & 65291 & 73 & $5.6295 \mathrm{E}+14$ & 106 & $4.8357 \mathrm{E}+24$ \\
\hline 41 & 130827 & 74 & $1.1259 \mathrm{E}+15$ & 107 & $9.67141 \mathrm{E}+24$ \\
\hline 42 & 261899 & 75 & $2.2518 \mathrm{E}+15$ & 108 & $1.93428 \mathrm{E}+25$ \\
\hline 43 & 524043 & 76 & $4.5036 \mathrm{E}+15$ & 109 & $3.86856 \mathrm{E}+25$ \\
\hline 44 & 1048331 & 77 & $9.0072 \mathrm{E}+15$ & 110 & $7.73713 \mathrm{E}+25$ \\
\hline 45 & 2096907 & 78 & $1.80144 \mathrm{E}+16$ & 111 & $1.54743 \mathrm{E}+26$ \\
\hline 46 & 4194059 & 79 & $3.60288 \mathrm{E}+16$ & 112 & $3.09485 \mathrm{E}+26$ \\
\hline 47 & 8388363 & 80 & $7.20576 \mathrm{E}+16$ & 113 & $6.1897 \mathrm{E}+26$ \\
\hline 48 & 16776971 & 81 & $1.44115 \mathrm{E}+17$ & 114 & $1.23794 \mathrm{E}+27$ \\
\hline 49 & 33554187 & 82 & $2.8823 \mathrm{E}+17$ & 115 & $2.47588 \mathrm{E}+27$ \\
\hline 50 & 67108619 & 83 & $5.76461 \mathrm{E}+17$ & 116 & $4.95176 \mathrm{E}+27$ \\
\hline 51 & 134217483 & 84 & $1.15292 \mathrm{E}+18$ & 117 & $9.90352 E+27$ \\
\hline 52 & 268435211 & 85 & $2.30584 \mathrm{E}+18$ & 118 & $1.9807 \mathrm{E}+28$ \\
\hline 53 & 536870667 & 86 & $4.61169 \mathrm{E}+18$ & 119 & $3.96141 \mathrm{E}+28$ \\
\hline 54 & 1073741579 & 87 & $9.22337 \mathrm{E}+18$ & 120 & $7.92282 \mathrm{E}+28$ \\
\hline 55 & 2147483403 & 88 & $1.84467 \mathrm{E}+19$ & 121 & $1.58456 \mathrm{E}+29$ \\
\hline 56 & 4294967051 & 89 & $3.68935 \mathrm{E}+19$ & 122 & $3.16913 \mathrm{E}+29$ \\
\hline 57 & 8589934347 & 90 & $7.3787 \mathrm{E}+19$ & 123 & $6.33825 E+29$ \\
\hline 58 & 17179868939 & 91 & $1.47574 \mathrm{E}+20$ & 124 & $1.26765 \mathrm{E}+30$ \\
\hline 59 & 34359738123 & 92 & $2.95148 \mathrm{E}+20$ & 125 & $2.5353 E+30$ \\
\hline 60 & 68719476491 & 93 & $5.90296 \mathrm{E}+20$ & 126 & $5.0706 \mathrm{E}+30$ \\
\hline 61 & $1.37439 \mathrm{E}+11$ & 94 & $1.18059 \mathrm{E}+21$ & 127 & $1.01412 \mathrm{E}+31$ \\
\hline 62 & $2.74878 \mathrm{E}+11$ & 95 & $2.36118 \mathrm{E}+21$ & 128 & $2.02824 \mathrm{E}+31$ \\
\hline 63 & $5.49756 \mathrm{E}+11$ & 96 & $4.72237 \mathrm{E}+21$ & & \\
\hline 64 & $1.09951 \mathrm{E}+12$ & 97 & $9.44473 \mathrm{E}+21$ & & \\
\hline
\end{tabular}

As we can see in Table2, if IP address size is 32-bit, the unsigned blocks are 11. But if we increase the size to 33-bit, this will expand the number of unsigned blocks to 267 . And so number of unsigned blocks grows exponentially as the address size increases until it reaches the value of (2.02824E+31) blocks for 128-bit address size. This number of blocks is equivalent to address space of (3.402822057984E+38) IP addresses.

\subsubsection{World population :( 1950 - 2050)}

The world population numbers issued by international programs which lists the world population for years from 1950 to 2050 [4] contains two parts: a short-term projection to 2050, together with historical population estimates back to 1950. Table 3 shows world population for each year. 
As we can see in the table 3, world population rose from 2,557,628,654 for year 1950 with growth rate about 1.5 percent per year up to $3,209,631,895$ with growth rate about 2.223 percent per year for year 1963, then growth rate started to decrease until it reached 0.468 for year 2049 with total population 9,339,454,816. For 13 years growth rate keeps increasing while the decreasing interval extends to 87 years and it is expected to keep decreasing after year 2050. Growth rate formula:

The projection of world population is performed using annual growth rate, where the annual growth rate is calculated for each year using the current and next year population. The growth rate formula for calculating the growth rate for any year is as following:

$\mathrm{r}(\mathrm{t})=[(\mathrm{P}(\mathrm{t}+1)-\mathrm{P}(\mathrm{t})) / \mathrm{P}(\mathrm{t})] * 100[4]$

Where:

$\mathrm{t}$ : year, $\mathrm{r}(\mathrm{t})$ : growth rate from midyear $\mathrm{t}$ to midyear $\mathrm{t}+1, \mathrm{P}(\mathrm{t})$ : population at midyear $\mathrm{t}$.

Table 3: Total Midyear Population for the World: 1950-2050 [4]

\begin{tabular}{|c|c|c|c|c|c|}
\hline Year & Ponulation & Year & Ponulation & Year & Ponulation \\
\hline 1950 & 2.557 .628 .654 & 1985 & 4.855 .387 .634 & 2020 & 7.628 .361 .509 \\
\hline 1951 & $2.594,919.657$ & 1986 & $4,939,332,441$ & 2021 & $7,701,503,831$ \\
\hline 1952 & 2.636 .732 .631 & 1987 & 5.025 .796 .394 & 2022 & 7.773 .787 .217 \\
\hline 1953 & $2.681,994,386$ & 1988 & $5,113,007,284$ & 2023 & $7,845,094,405$ \\
\hline 1954 & 2.730 .149 .884 & 1989 & 5.199 .760 .484 & 2024 & 7.915 .343 .988 \\
\hline 1955 & $2,782,001,154$ & 1990 & $5,287,166,778$ & 2025 & $7,984.471 .678$ \\
\hline 1956 & 2.835 .182 .293 & 1991 & 5.370 .142 .696 & 2026 & 8.052 .555 .718 \\
\hline 1957 & 2.891 .211 .793 & 1992 & 5.455 .057 .523 & 2027 & 8.119 .653 .594 \\
\hline 1958 & 2.947 .979 .287 & 1993 & 5.537 .583 .721 & 2028 & 8.185 .704 .067 \\
\hline 1959 & 3.000 .544 .325 & 1994 & 5.618 .516 .091 & 2029 & 8.250 .680 .127 \\
\hline 1960 & $3,042,828,380$ & 1995 & $5.699,516,291$ & 2030 & $8,314,556,118$ \\
\hline 1961 & 3.083 .799 .968 & 1996 & 5.779 .912 .412 & 2031 & 8.377 .397 .726 \\
\hline 1962 & 3.139 .919 .051 & 1997 & 5.858 .582 .659 & 2032 & 8.439 .275 .616 \\
\hline 1963 & 3.209 .631 .895 & 1998 & 5.936 .039 .484 & 2033 & 8.500 .179 .665 \\
\hline 1964 & 3.280 .981 .862 & 1999 & 6.013 .121 .531 & 2034 & 8.560 .091 .213 \\
\hline 1965 & 3.350 .186 .115 & 2000 & 6.089 .810 .661 & 2035 & 8.618 .975 .745 \\
\hline 1966 & 3.420 .416 .498 & 2001 & 6.166 .582 .980 & 2036 & 8.676 .879 .132 \\
\hline 1967 & $3,490,051,163$ & 2002 & $6,243,351,444$ & 2037 & $8,733,854,464$ \\
\hline 1968 & 3.562 .007 .503 & 2003 & 6.319 .822 .330 & 2038 & 8.789 .875 .606 \\
\hline 1969 & 3.636 .825 .800 & 2004 & 6.396 .726 .866 & 2039 & 8.844 .910 .290 \\
\hline 1970 & 3.712 .338 .708 & 2005 & 6.473 .525 .274 & 2040 & 8.898 .921 .851 \\
\hline 1971 & 3.789 .941 .225 & 2006 & 6.551 .256 .997 & 2041 & 8.951 .939 .549 \\
\hline 1972 & 3.866 .158 .404 & 2007 & 6.629 .668 .134 & 2042 & 9.003 .999 .017 \\
\hline 1973 & 3.941 .664 .971 & 2008 & 6.708 .196 .774 & 2043 & 9.055 .061 .670 \\
\hline 1974 & 4.016 .159 .586 & 2009 & 6.786 .381 .274 & 2044 & 9.105 .083 .460 \\
\hline 1975 & 4.088 .619 .689 & 2010 & 6.863 .770 .931 & 2045 & 9.154 .029 .673 \\
\hline 1976 & 4.159 .715 .844 & 2011 & 6.940 .712 .355 & 2046 & 9.201 .933 .427 \\
\hline 1977 & 4.231 .636 .519 & 2012 & 7.017 .543 .964 & 2047 & 9.248 .825 .693 \\
\hline 1978 & 4.303 .675 .842 & 2013 & 7.095 .217 .980 & 2048 & 9.294 .674 .737 \\
\hline 1979 & 4.378 .583 .227 & 2014 & 7.172 .800 .105 & 2049 & 9.339 .454 .816 \\
\hline 1980 & 4.450 .924 .299 & 2015 & 7.250 .104 .524 & 2050 & 9.383 .147 .855 \\
\hline 1981 & 4.533 .807 .914 & 2016 & 7.327 .047 .197 & & \\
\hline 1982 & 4.613 .830 .568 & 2017 & 7.403 .533 .981 & & \\
\hline 1983 & 4.694 .935 .057 & 2018 & 7.479 .340 .547 & & \\
\hline 1984 & 4.773 .643 .742 & 2019 & 7.554 .324 .522 & & \\
\hline
\end{tabular}

\subsubsection{IP exhaustion date prediction}

We can obviously note that the relation between IP address consumption and world population is proportional. This relation can be strongly proved if we take a look at the table of annual IP addresses consumption and the table of annual world population. So, we will use the population projection to predict the exhaustion date for several IP address sizes.

First of all, we will derive an equation that we will use in the prediction of IP addresses consumption for the next year depending on IP address consumption for the current year. In order to do that, we will use population growth rate equation to generate predicted needed address space for years after 2013. The following is IP address consumption prediction equation that we derived assuming that IP consumption growth rate is equal to population growth rate:

$\operatorname{IP}(\mathrm{t}+1)=[(\operatorname{IPr}(\mathrm{t}) * \operatorname{IP}(\mathrm{t})) / 100]+\mathrm{P}(\mathrm{t})$

Where:

IP (t): allocated IP addresses at year $t$, IP $(t+1)$ : allocated IP addresses at year $t+1$,

$\operatorname{IPr}(\mathrm{t})$ : growth rate from midyear $\mathrm{t}$ to midyear $\mathrm{t}+1$.

After that, we will use equation (2) to generate expected unsigned IP blocks for each years starting from 2013 to the year corresponding to the address space that 128-bit IP address can provide. In order to do that, we need growth rate of IP address consumption for each year in that range. If we take a look at table 2, we will see how the growth rate of population started decaying from year 1967 and kept decaying to the last year of projection and if we made a long- term projection it is expected to decay also to the last year of projection. In other word, the growth rate of the year 2050 is expected to be the maximum growth rate until any year the long term projection will end with. So, for our 
approximation we will use the growth rate of year 2050 as an approximation for IP growth rate consumption for any year after 2050. This assumption is not the ideal assumption, but this paper tries to predict the expected IP address consumption from world population point of view.

\subsubsection{Expected allocated IP address blocks (2013 - 16285)}

In this part, we will focus on generating the expected needed IP address blocks for years form 2013 until 16285 (figure 3 ) as a first step. So we can use these numbers together with Table1 as a base for the generation of the expected exhibition date for different IP address sizes. We accomplished this task by matching the address space that each address size can provide with the expected needed block address for each year.

The chart in figure 3 represents the relationship between the number of expected allocated addresses and the year for that allocation. The duration that this figure covers is 14272 years which could be considered as a long term prediction. The importance of this kind of study is based on developing a solution for IP address exhibition for thousands and thousands of years.

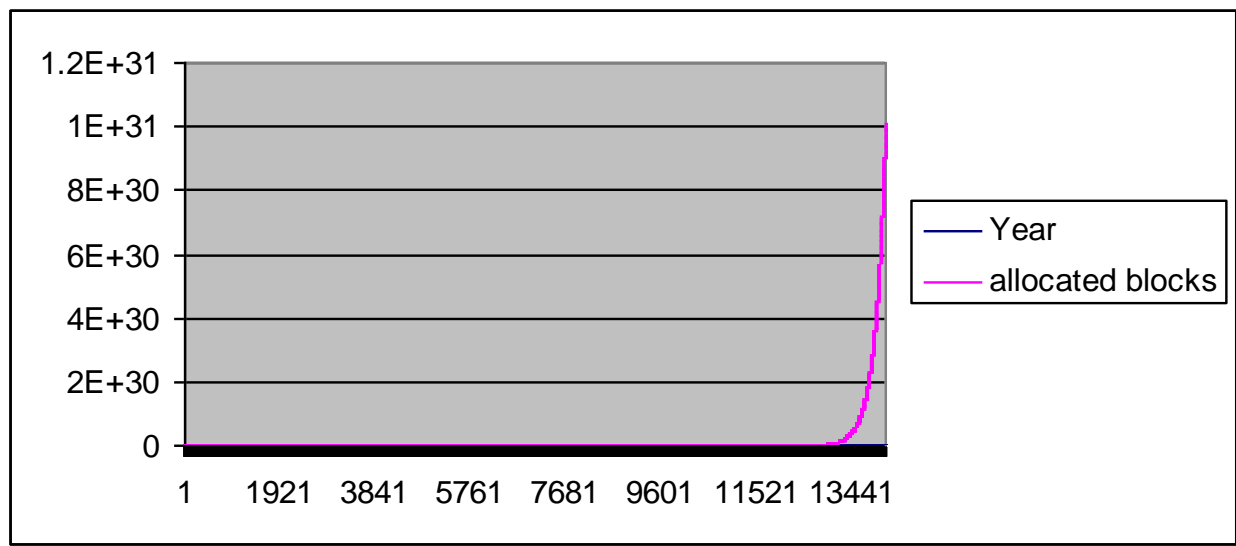

Fig. 3: Curve represent number of expected allocated IP address blocks in years from 2013 to 16136 . Where the number of allocated blocks for the first 1000 years after 2013 increases slowly compared with allocated blocks from 4000 to 16136 which increases from 2486934 to $1.01047 \mathrm{E}+31$ blocks.

As we can see in the above figure 3 the number of expected allocated IP address blocks is exponentially increasing towards $1.01047 \mathrm{E}+31$ blocks in year 16136. Also we can note that the number of allocated blocks for the first 1000 years after 2013 increases slowly compared with allocated blocks from 4000 to 16136 which increases from 2486934 to 1.01047E+31 blocks.

\subsubsection{Exhibition date for IP address of size $N$}

Now, we will use the expected allocated IP address blocks prediction together with available address space for IP address of size $\mathrm{N}$ (Table1) to predict the exhaustion of the available address space for an IP address range from 32-bit to 128-bit. (For example, the study predicts that the unassigned address for the 32-bit address space will be exhausted by 2017). In the same way, we will predict when the address space for each IP address size will be exhibited. To cover the range from 32-bit to 128-bit, we picked an interval of 8 bits length between two consecutive addresses size. Figure 4 shows the prediction for this range of address size.

As shown in Figure 4, the number of years ranging between 32-bit and 128-bit of IP address that can be accommodated is growing up starting from year 2017 for address size 32-bit until the year of 16285 for address size 128-bit with difference equal to 14268 years.

For 40-bit IP address size the exhibition date will be in year 3221, and for the next IP address size in the figure the date becomes 4408. After that, the exhibition date 56-bit address size will offer is 5596 which means 1188 years more than the previous one and this number increases for 64-bit address size to 2376 years. Then, for 72 -bit address size the exhibition date will be 7971 and for 80-bit it will be 9159 which will be increased to 10346 for 88 -bit address size. This increase in predicted exhibition date continues for 96-bit to be 11534 and so on. Finally, for 128-bit address size the exhibition date will jump all the way to 16285 .

If we reduce the IPv6 address size from 128-bit to 64-bit, this size can accommodate 18,446,744,069,599,100,000 IPv6 addresses and exhibition date will be at year 6748, which is more than 4000 years from now. So, why should we stick to 128-bit IP address size while 64-bit address size provides reduction of basic header IPv6 packet overhead up to $40 \%$ of the packet header total size which is considered a significant reduction that will improve the overall IPv6 performance for at least 4000 years. 


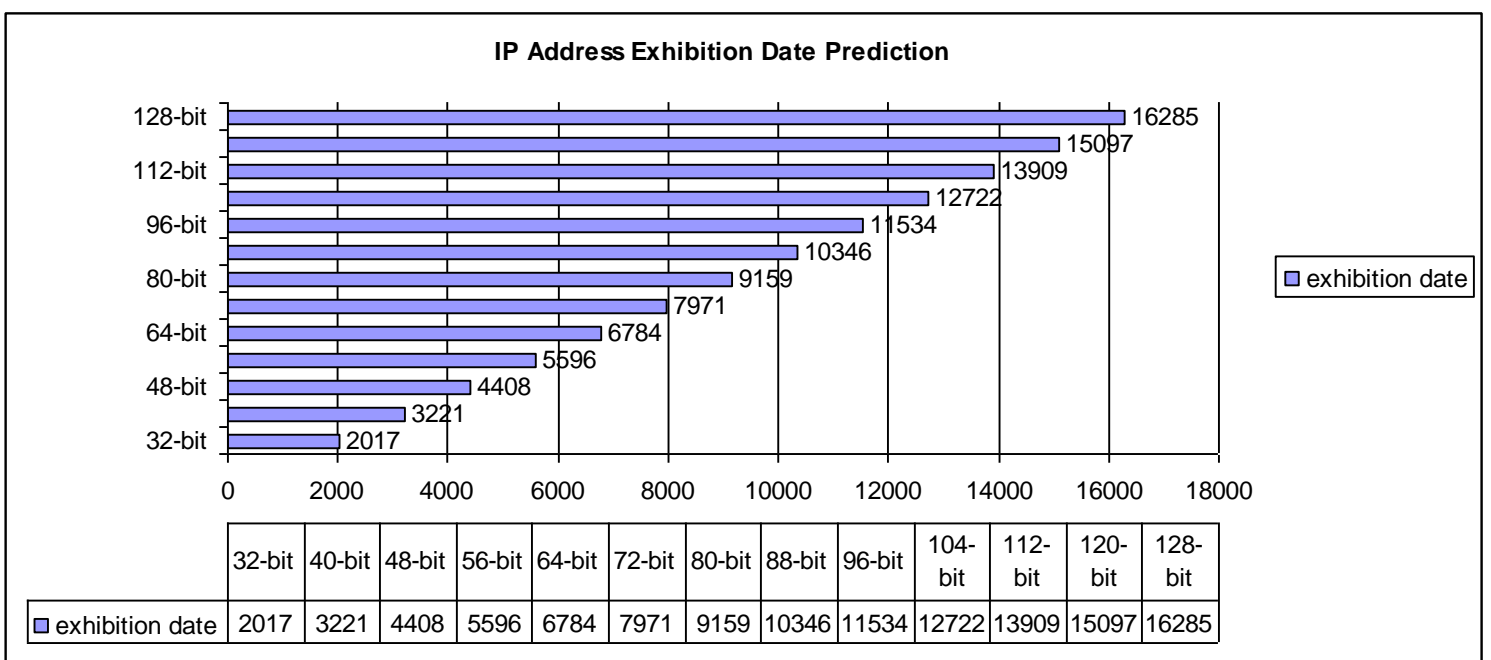

Fig. 4: prediction of exhaustion date for the available address space for an IP address range from 32-bit to 128-bit. To cover the range from 32-bit to 128-bit, we picked an interval of 8 bits length between two consecutive addresses size.(ex: exhaustion date for 32-bit address is 2017)

\section{Merging IP exhibition prediction studies}

In the first study that we have presented it in our paper we can clearly see that exhibition date for IP addresses size from 32-bit to 48-bit are less than exhibition date for its corresponding sizes in the second study, for larger address sizes the difference between the two studies decreases until the second study exhibition date starts to be larger than its corresponding address sizes in the first study then this difference starts to grow exponentially as the size increases until it reached 1237365 years for address size of 64-bit which is an extremely significant difference. And so we will generate new IP address exhibition date prediction considering both prediction studies as a base for our prediction with dynamic percentage impact for each study $(0 \%$-- 100\%, $10 \%$-- 90\%, $20 \%$-- 80\%, 30\% -- 70\%, $40 \%$-- $60 \%, 50 \%$-$50 \%, 60 \%--40 \%, 70 \%--30 \%, 80 \%--20 \%, 90 \%--10 \%$ and $100 \%--0 \%$ ).

Table 4: Exhibition date prediction for address sizes 32-bit, 40-bit, 56-bit and 64-bit involving the first study and the second study in this paper, each time with different factor ratio for each one.

\begin{tabular}{|c|c|c|c|c|c|}
\hline \multirow{2}{*}{ Study1\%---Study2\% } & \multicolumn{5}{|c|}{ IP Address Size } \\
\hline & 32-bit & 40-bit & 48-bit & 56-bit & 64-bit \\
\hline $0 \%---100 \%$ & 2017 & 3221 & 4408 & 5596 & 6784 \\
\hline $10 \%---90 \%$ & 2016.5 & 3128.5 & 4322 & 12803.9 & 130520.5 \\
\hline $20 \%$ & 2016 & 3036 & 4236 & 20011.8 & 254257 \\
\hline $30 \%$---- $70 \%$ & 2015.5 & 2943.5 & 4150 & 27219.7 & 377993.5 \\
\hline $40 \%---60 \%$ & 2015 & 2851 & 4064 & 34427.6 & 501730 \\
\hline $50 \%---50 \%$ & 2014.5 & 2758.5 & 3978 & 41635.5 & 625466.5 \\
\hline $60 \%$ & 2014 & 2666 & 3892 & 48843.4 & 749203 \\
\hline $70 \%$---- $30 \%$ & 2013.5 & 2573.5 & 3806 & 56051.3 & 872939.5 \\
\hline $80 \%$---- $20 \%$ & 2013 & 2481 & 3720 & 63259.2 & 996676 \\
\hline $90 \%---10 \%$ & 2012.5 & 2388.5 & 3634 & 70467.1 & 1120413 \\
\hline $100 \%---0 \%$ & 2012 & 2269 & 3548 & 77675 & 1244149 \\
\hline AVG & 2014.5 & 2756 & 3978 & 41636 & 625467 \\
\hline
\end{tabular}

In the above table the exhibition dates for IP address sizes starts from 32-bit address then we add 8-bit each time until the size reaches 64-bit. Each prediction has been made depending on the first study with specific percentage and the second study with percentage $=(\% 100-$ study 1 percentage $)$. All percentage are listed in the first column.

The aim of the above table is to involve both studies in prediction of IP exhibition date, each time with different factor ratio for each study. So in the first row all predictions depend purely on the second study then in the second row it depend 90 percent on the second study and 10 percent on the first study, for each row the percentage of the first study factor increases by 10 percent, at the same time the percentage of the second study factor decreases by 10 percent than its previous row until it depends purely on the first study. In the last row we calculate the average exhibition date using all factor ratios for each address size.

Exhibition dates are decreasing for IP address sizes 32-bit, 40-bit and 48-bit as we go down in each column where the factor of the first study increases and the factor of the second study decrease while for both address sizes 56-bit and 64bit exhibition dates increase. Also average exhibition date started from 2014.5 for address size 32-bit then growth 
exponentially all the way until it reaches 625467 for address size 64-bit which is the new proposed address size this paper is recommended.

\section{Conclusion}

This paper explained the redundancy of waste space that is reserved to IPv6 source and destination addresses in IPv6 standard packet large address size that occupies $80 \%$ of IPv6 basic packet header total size. It is concluded that it should be reduced to make that possible, we developed three studies. The first study is based on other study that was developed in 2005 by Geoff Haston. Our study predicts that exhibition date for each IP address size starting by 32 bit and increments this size 4 bits at a time until we reach 64 bits size. We explained that there is no need to expand this size any more. The second study was made based on IPv4 address report generated on 30-Jan-2013. The remaining addresses in RIR pool and world population projection growth rate include the derivation of new formula that predicts IP Address consumption. On the other hand, it shows that choosing 64-bit address size will solve the exhaustion problem. At the same time this size reduces the overhead in IPv6 packet basic header by $40 \%$. Our study predicts that exhibition date for each IP address size starting by 32-bit and incrementing this size 8 bits at a time until we reach 128bits size. Finally we used both studies to develop a third one which depends on both studies by generating a table that reflects a variable effect for each study in each row then we generate the average of overall generated effects. The reason for developing the third study is due to the significant difference between the predicted exhibition dates for several suggested address sizes. So we came up with this study based on the first two studies in order to get more accurate prediction.

\section{References}

[1] Geoff Huston, "IPv4 Address Report", Online: http://www.potaroo.net/tools/ipv4/index.html.

[2] Geoff Huston APNIC, "IPv4 Address Utilization", Online: http://www.potaroo.net/papers/2005-03-ipv4.pdf, March 2005.

[3] Patrick Grossetete, Ciprian P. Popoviciu, Fred Wettling, "Global IPv6 Strategies: From Business Analysis to Operational Planning" Online: http://media.techtarget.com/searchNetworking/downloads/IPv4_or_IPv6.pdf, 1st Edition, chapter 2, pp. 18-53, May 15, 2008.

[4] World Population, Online: http://www.census.gov/population/international/data/worldpop/table population.php Source: U.S. Census Bureau International Database.

[5] Charles M. Kozierok, "TCP/IP GUID A COMPREHENSIVE, And ILLUSTRATED INTERNET PROTOCOLS REFERENCE", Online: http://Nostarch.Com/Download/Tcpip_Ch25.Pdf, Chapter 25, Pp.373-381, and October 2005.

[6] Doglase E. Comer, Department of Computer seines, Purdue University, West Lafayette, "Internetworking with TCP/IP Principles, Protocols and Architectures", Fourth Edition, Volume 1, Chapter 7, pp. 95-107, 2000.

[7] Microsoft Corporation, Windows server 2008, "Introduction to IP version 6", Online: http://download.microsoft.com/download/e/9/b/e9bd20d3-cc8d-4162-aa60-3aa3abc2b2e9/IPv6.doc, Jan 2008.

[8] IPv6 Headers", Online: http://www.cu.ipv6tf.org/literatura/chap3.pdf, chapter 3, pp. 40-55, Des 121997. 